\title{
La historia de la teoría electroquímica y su contribución a la promoción de la explicación científica en la química escolar
}

\author{
The story of the electrochemical theory and their \\ contribution to the promotion of scientific \\ explanation school chemistry
}

Artículo recibido: febrero de 2011

Artículo aceptado: Junio de 2011

Johanna Camacho González*

\begin{abstract}
Resumen
Esta investigación presenta los principales hallazgos referentes a ¿cómo se construyen las explicaciones científicas acerca de la Teoría Electroquímica en las clases de Educación Media? La investigación se sustenta en la actual necesidad de promover la explicación como una competencia científica y, en el uso de la historia de la ciencia, como área metacientífica que contribuye favorablemente a dicha necesidad. Este artículo presenta resultados de una investigación que se realizó en Santiago de Chile, a través de un estudio de casos colectivo, en el cual se pudieron evidenciar dos secuencias diferentes para la construcción de las explicaciones mediante la enseñanza de la teoría electroquímica.
\end{abstract}

\section{Palabras clave}

Explicación científica, competencias científicas, teoría electroquímica, historia de la química.

\begin{abstract}
This research presents the main findings regarding how are scientific explanations about the theory Electrochemistry in High School? The research is based on the current need to promote the scientific explanation as a scientific competence and the use of history of science as an area metascientific that can contributing positively to this need. This study was conducted in Santiago de Chile through a collective case study, which was evident two different sequences for the construction of explanations through the teaching of the electrochemical theory.
\end{abstract}

\section{Keywords:}

Scientific explanation, scientific competencies, electrochemical theory, history of Chemical.

* Universidad de Chile. Facultad de Filosofía y Humanidades. Departamento de Estudios Pedagógicos. Área de Didáctica de las Ciencias., Santiago de Chile jpcamach@uchile.cl. 


\section{Introducción}

A pesar del gran intento de los últimos años por sugerir cambios curriculares, por ejemplo en Chile el Ajuste Curricular de Ciencias (MINEDUC, 2009), que incorporen los propósitos actuales de la educación científica a través de la promoción y desarrollo de competencias científicas, aún se evidencian indicadores que dan cuenta de una crisis en la enseñanza y aprendizaje de la química. Lo anterior se manifiesta, según Izquierdo (2004), en las opiniones desfavorables de quienes, ya mayores, recuerdan esta materia como algo incomprensible y aborrecible; también en la falta sostenida de personas que desean tener cursos optativos de esta área científica y en la disminución sistemática y ascendente de estudiantes que escogen la química como carrera profesional, particularmente en el área docente.

Según esta investigadora se establecen, entre otras, dos posibles causas de esta crisis: primero, la enseñanza desde una perspectiva demasiado dogmática, alejada de las finalidades y valores de las y los estudiantes; y, en segundo lugar, quizás la educación química se ha visto sólo desde la perspectiva de la enseñanza de ideas teóricas sin explicar suficientemente a qué tipo de intervención se refieren, por lo que la práctica se convierte para el estudiantado en un ejercicio irracional, conectando conocimientos que no son comprendidos ni útiles. Esta crisis también queda en evidencia en las afirmaciones del profesorado de química, quienes han señalado que la enseñanza de esta ciencia se caracteriza por la dificultad en aplicar los conocimientos: usualmente los y las estudiantes creen que no les sirve para explicar el mundo en el que viven.

Un ejemplo específico de lo que comenta Izquierdo (2004) se puede evidenciar a través de la enseñanza y aprendizaje de la electroquímica, caracterizada como una de las temáticas más difíciles de enseñar y aprender en la química escolar (Davies, 1991; Griffths, 1994: De Jong, Acampo \& Verdonk, 1995; Níaz \& Chancón, 2003; Özkaya, 2002). Esta dificultad radica, según De Jong \& Treagust (2002), en aspectos conceptuales sobre la dependencia mutua de las reacciones de oxido reducción, el significado del número de oxidación, el proceso de transferencia de electrones, la carga del ánodo y el cátodo en las pilas electroquímica; así como en aspectos procedimentales, en relación a la identificación de reactantes como agentes oxidantes o reductores, ecuaciones químicas de oxido reducción y el ánodo o cátodo en una pila.

Otro indicador de esta situación de crisis, con sustento empírico, lo constituyen los resultados de Chile en las pruebas internacionales PISA, donde, a pesar de aumentar significativamente en la escala general de 438 puntos en PISA 2006 (OCDE, 2006), a 447 puntos en PISA 2009 (OCDE, 2010), se presentan diferencias significativas en los resultados de cada competencia evaluada. Con base en los resultados de PISA 2006, cuyo énfasis fue Competencias Científicas, Gubler y Williamson (2009) realizaron un análisis acerca de cada una de las competencias científicas, estructurado a partir de tres parámetros: a) Explicar fenómenos científicamente, b) Identificar cuestiones científicas y c) Utilizar pruebas científicas (OCDE, 2006). En una escala general (que combina las tres competencias), los y las estudiantes en Chile obtuvieron un puntaje promedio de 438 puntos, significativamente inferior al promedio del conjunto de los países OCDE. En cuanto a las competencias evaluadas, el promedio obtenido en la competencia explicar fenómenos científicamente fue de 432 puntos; identificar cuestiones científicas fue de 444 puntos y en utilizar pruebas científicamente fue de 440 puntos. En estos resultados se aprecia que la competencia explicar fenómenos cientificamente es la que presenta mayores dificultades para los y las jóvenes de Chile.

Estos antecedentes han llevado a repensar la enseñanza de las ciencias en general y de la química en particular, en donde se sugiere, además de la revisión del currículo, considerar la calidad del profesorado y cómo a través de su práctica se promueven las competencias científicas en sus estudiantes. 


\section{La explicación científica.} Una competencia científica

La idea de la explicación científica ha sido una preocupación teórica importante durante la historia y epistemología de la ciencia (Hempel 1965; Giere, 1992; Rosenberg, 2000); así, han surgido investigaciones en el campo de la didáctica de las ciencias que buscan profundizar al respecto en las aulas de ciencias, estableciendo diferentes diseños a fin de comprender el papel de la explicación (junto con la argumentación y justificación) en la metodología científica, la teoría de la elección, cambiar la teoría, y la comunicación de la ciencia (Izquierdo \& Adúriz-Bravo, 2009). Sanmartí e Izquierdo (1998) consideran que la explicación es una actividad fundamental porque "lo que interesa es la comprensión, el establecimiento de relaciones, la negociación de significado en el aula" (184). Wartofsky (citado en Sanmartí e Izquierdo, 1998) señala además que "explicar algo es haber llegado a entenderlo de tal manera que uno sea capaz de hacer que otro lo entienda". En este sentido, Welsh (2002) afirma la importancia de hacer hincapié en la estructura de las explicaciones en la enseñanza de la química, en vista de que esta ciencia se ha construido a través de explicaciones científicas que por lo general ayudan al estudiantado a comprender mejor lo que aprende. Él propone que muchas explicaciones correlacionan diferentes partes, estructura a la que ha denominado $F a C T s$, (Hechos) donde la $F$ significa «forma»; $C$, su «composición» y T la «teoría». Entonces,

[...] una explicación científica tiene cuatro partes de acuerdo a los FacTs que se pretenden establecer. Cuando una "teoría" (Parte 1) correspondiente a la química es aplicada a la "composición" (Parte 2) y "forma"(Parte 3) de uno o más átomos de interés, entonces las conclusiones o deducciones resultantes de la aplicación de lo que la teoría debe ser coherente con los "hechos" (Parte 4) observados" (Welsh, 2002: 94).

Sin embargo, existen diferencias entre identificar la explicación como una habilidad cognitivo-lingüística indispensable para «hablar y escribir para aprender ciencias», y como una «capacidad relacionada con competencias científicas». Con respecto a la explicación como habilidad cognitivo-lingüística,
Jorba (2000) propone que ésta consiste en "producir razones o argumentos de manera ordenada. Establecer relaciones entre las razones y argumentos que lleven a modificar un estado de conocimiento" (43).

Además de lo anterior, Sanmartí e Izquierdo (1998) precisan otros atributos necesarios para la construcción de una explicación científica, tales como:

- Estructurar el texto de una manera expositiva: con un inicio, un desarrollo y una conclusión.

- Desarrollar una situación inicial mostrando los hechos nuevos que después permiten llegar a una conclusión.

- Relacionar los hechos nuevos y los conocidos de manera fácil de aceptar, porque se ha aplicado a situaciones analógicas; la novedad está en las informaciones concretas que se ofrecen o en las conexiones entre las mismas, pero no en los dos aspectos a la vez. En general estas relaciones son causa-efecto.

- Seleccionar hechos relevantes e interesantes; el alumnado puede ser muy creativo al ofrecer una explicación y elaborar textos parecidos a los literarios.

- Situar toda la explicación en un contexto temático bien caracterizado.

- Ofrecer una nueva perspectiva que permite hacer inferencias, siempre a nivel factual de "cosas que pueden pasar o no pueden pasar".

Como competencia científica, la explicación ha llamado la atención de la OCDE, como una capacidad importante de desarrollar en la educación científica. Así, ésta ha sido "aplicar el conocimiento de la ciencia a una situación determinada; describir o interpretar fenómenos científicamente y predecir cambio e identificar las descripciones, explicaciones y predicciones apropiadas" (OCDE, 2006: 30).

En general se observa, a través de la literatura especializada, que la explicación científica está asociada con la capacidad que tiene el estudiantado de construir relaciones teóricas coherentes sobre distintos fenómenos naturales; un proceso que implica proponer relaciones nuevas y elaborar conclu- 
siones que suponen modificaciones de un estado de conocimiento. No obstante, en el desarrollo de la competencia también se contempla la complejidad de estos procesos dentro del pensamiento de la persona que construye su competencia y cómo ésta toma decisiones sobre lo que debe saber hacer, saber conocer, saber comunicar y saber ser. Así, la "Persona competente debería ser capaz además de representar el mundo en el que convive con teoría científica, tomar decisiones que le permitan intervenir y transformarlo de manera autónoma, autorregulada, creativa e independiente, según sus necesidades y motivos, sus objetivos y acciones, los medios y operaciones que utiliza para enfrentarse a una situación problemática" (Camacho, 2010: 80).

De esta manera, según Camacho (2010), la explicación científica se puede caracterizar como una competencia científica (Figura 1), esencial para la actividad química escolar, ya que la persona competente construye y reconstruye sus explicaciones a través de procesos de modelización que demuestran la comprensión conceptual que tiene de un fenómeno mediante la teoría científica. Para lo cual desarrollará diferentes procesos relacionados con sus conocimientos, el contexto, su ser y su actuar. Esta visión de la explicación científica sugiere, además, como un aspecto relevante el rol significativo y personal, metacognitivos, afectivos y emocionales, que le otorgue la persona durante la construcción de su competencia.

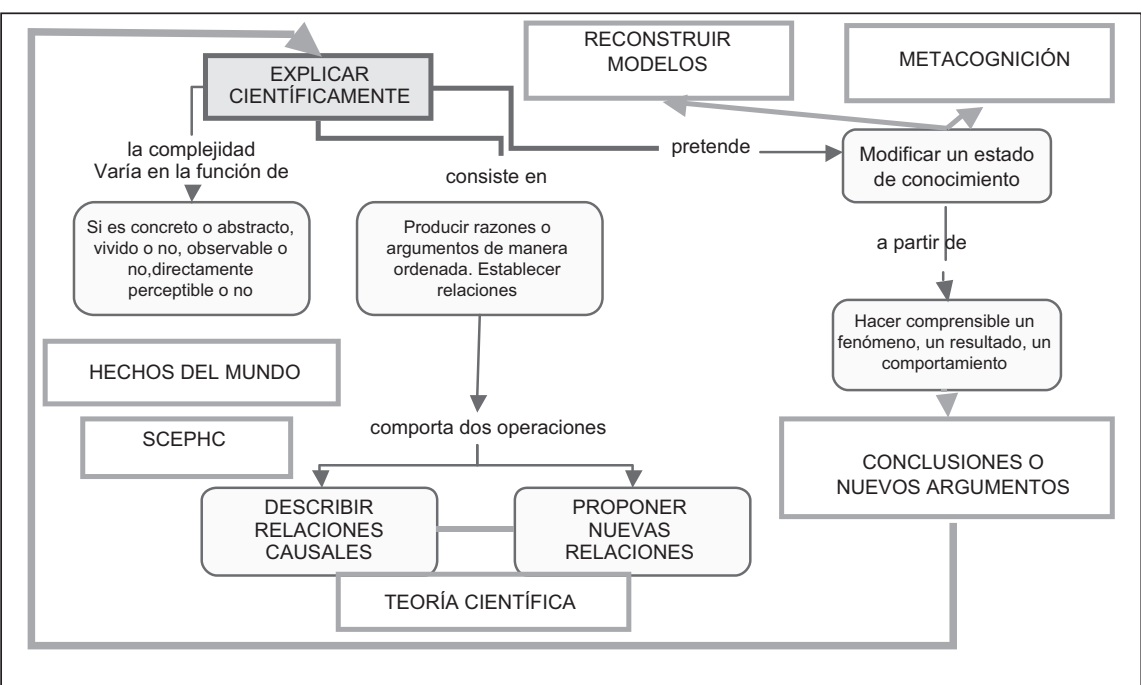

Figura 1. La explicación como competencia científica

\section{Historia de la ciencia para la promoción de la explicación científica}

Los propósitos de la actividad química escolar están relacionados con la formación de personas competentes en dicho campo; así, esta actividad debe establecer una imagen de ciencia menos dogmática y más relacionada con la actividad de hombres y mujeres para la resolución de sus problemas cotidianos. De esta forma, la actividad química escolar se transforma en un espacio de discusión y construcción de explicaciones del mundo, a través del estudio de "situaciones científicas escolares problematizadoras" (Camacho \& Quintanilla, 2008), que se caracterizan por ser actividades auténticas, innovadoras y similares a situaciones que se desarrollan en los contextos científicos reales; pero también significativas para el estudiantado que aprende; relevantes para la disciplina científica que se enseña; en síntesis, actividades que promueven ejercicios reflexivos, frente a los cuales el estudiante se enfrenta empleando procesos de modelización científica.

El objetivo de la incorporación de la historia de la ciencia en la actividad química escolar, no consiste en transformar al estudiantado o docentes en historiadores de la ciencia, sino que se orienta a promover y desarrollar competencias científicas que permitan un conocimiento y comprensión sobre la actividad química (Camacho, 2010). Así, a través de la lectura e interpretación de fuentes primarias, la réplica de experimentos, la propuesta de debates históricos en el aula, entre otras actividades, el estudiantado es capaz de comprender cómo se construye el conocimiento científico; cómo se elaboran las explicaciones científicas; explicar por qué una teoría científica se desarrolló con más éxito que otra; qué hizo posible que evolucionará dicho conocimiento; cómo era el contexto en el qué se desarrolló y

Fuente: Modificado de Jorba, 2000: 43. 
cómo influyó ese nuevo conocimiento en dicho momento; cómo se enfrentaban las personas a los problemas teóricos, experimentales y profesionales; cómo argumentaban sus ideas científicas; qué instrumentos disponibles hicieron posible el desarrollo y la consolidación de determinados conceptos; qué les permitieron concluir en su momento; cómo estas son evaluadas por las comunidades de especialistas, entre otros aspectos relacionados con la ciencia y el género, la cultura material, la socialización y popularización de la ciencia, etc. Estos aspectos, según Gooday, et al (2008), son quizá más eficaces que simplemente aprender y reproducir el contenido de libros de texto de ciencias y las rutinas de laboratorio, ya que permiten apreciar el carácter incompleto y la falibilidad de los modelos y teorías, así como una comprensión amplia que caracterizan la complejidad de cómo la ciencia cambia, modificando la imagen estática que muestran los libros de texto (Camacho y Martínez, 2003; Camacho, 2005; Camacho, Gallego, Pérez, 2007).

\section{Tendencias y obstáculos en la enseñanza- aprendizaje de la teoría electroquímica}

La electroquímica se ha caracterizado como una de las temáticas más complejas de enseñar y aprender en la química escolar (Davies, 1991; Griffths, 1994: De Jong, Acampo \& Verdonk, 1995; Níaz \& Chancón, 2003; Özkaya, 2002). Tradicionalmente la enseñanza de este concepto químico en las aulas y a través de los libros de texto, según De Jong \& Treagust (2002), ha estado divido en los procesos de óxido reducción y las pilas electroquímicas o celdas galvánicas, en particular la Pila de Daniell. Además, existe poca evidencia empírica, comparada con otras temáticas químicas, que relacionen las dificultades de aprendizaje de los estudiantes, señalen los principales obstáculos en la enseñanza y presente nuevas propuestas de innovación en el aula.

En un estudio detallado, De Jong y Treagust (2002) señalan con respecto a las dificultades de las reacciones de óxido reducción los siguientes aspectos: a) conceptuales entorno a la dependencia mutua de las reacciones de óxido reducción, el significado del número de oxidación, el proceso de transferencia de electrones, la carga del ánodo y el cátodo en las pilas electroquímica. A partir de allí se señalan los obstáculos que existen para comprender la conducción de la electricidad, la neutralidad eléctrica en la electricidad, identificar el ánodo y cátodo en una pila y sus respectivas cargas; b) procedimentales, en relación a la identificación de reactantes como agentes oxidantes o reductores y de ecuaciones químicas, en términos de óxido reducción. En cuanto a las dificultades relacionadas con las pilas electroquímicas se propone la dificultad de predecir los productos y la magnitud de diferentes potenciales en las celdas galvánicas. Además del componente instrumental y algorítmico, al cual muchas veces se reduce su enseñanza, favoreciendo así actividades hacia la memorización de los números de oxidación, el balanceo de ecuaciones y la resolución de ejercicios cuantitativos, con una débil comprensión conceptual.

La complejidad de la enseñanza y aprendizaje de la electroquímica radica en las prácticas experimentales asociadas, provenientes de diferentes áreas científicas como la fisiología, la física, la química y la matemática; además, en el uso de múltiples definiciones y modelos, los cuales están relacionados con la propia evolución de los diferentes conceptos científicos.

Como afirma Izquierdo (2004), "las ciencias son el resultado de una actividad humana muy compleja sustentada como cualquier otra actividad humana en pluralidad de sistemas de valores; nunca fueron ni son ahora neutras" (116). A continuación se presenta un ejemplo en la evolución de los conceptos de oxidación y reducción en los distintos períodos del desarrollo de la Teoría Electroquímica.

[...] el campo de las reacciones redox ha variado en los distintos períodos de la historia de la química, a finales del S. XVIII, los términos de oxidación y reducción fueron presentados por el científico francés Lavoisier en relación con sus estudios de combustión. Él utilizó estos términos para describir la reacción del oxígeno con otras sustancias en particular, y la eliminación de oxígeno de los productos forma- 
dos. En el principio del siglo XIX, el alemán científico Liebig propuso otra definición, desde sus estudios sobre el proceso deshidrogenación de alcoholes a aldehídos. Él prefirió definir la oxidación y reducción con respecto a la pérdida y ganancia de hidrógeno. En el comienzo del S. XX, el científico estadounidense Lewis, con su teoría electrónica de la valencia, propone definir una reacción redox como una combinación de dos medias reacciones incluyendo la transferencia de electrones. Más tarde, el científico estadounidense Latimer introdujo el término de número de oxidación como una noción completamente formal, describir una reacción redox como una reacción vinculada al cambio de los números de oxidación. (De Jong \& Treagust, 2002: 318).

Enfocándose en la relación entre el desarrollo conceptual de la teoría electroquímica y los obstáculos y dificultades que se presentan en la enseñanza-aprendizaje, se estudió a continuación la evolución histórica de la teoría electroquímica entre 1800 y 1853.

\section{Acerca de la evolución histórica de la Teoría Electroquímica}

Según Grapí i Vilumara (2008), "el descubrimiento de la pila eléctrica de Volta al principio del S. XIX va a abrir a los químicos las fronteras de un nuevo territorio que va a resultar fuertemente fructífero en diversos campos". Si bien en el siglo XVIII la preocupación de la comunidad científica, con respecto a la electricidad, estaba enfocada hacia la producción de la misma a través de medios artificiales (la fricción) o naturales (pez torpedo o la contracción muscular), es hasta el siglo XIX que se empieza a evidenciar una relación entre la producción de electricidad mediante la descomposición química.

A través del experimento de Galvanic con las ancas de rana (Galvanismo), se tiene en cuenta la acción de los metales, hecho que aprovecharía favorablemente Volta para la elaboración de su Pila y la explicación de la producción de electricidad en relación a los fluidos. Sin embargo, Davy es quien va a establecer una relación entre la electricidad y la descomposición química de las sustancias, otorgando un significado importante en relación a la afinidad o no de las sustancias según su naturale- za eléctrica. Los aportes de otros científicos como Oersted, Ampére y Liebig van configurando la teoría electroquímica que posteriormente Berzelius logra interpretar en función de las proporciones de materia que se combinan y de los flujos eléctricos que estaban implicados "Es claro que la misma causa, que produce las combinaciones o las descomposiciones como la pila eléctrica, deben cooperar también en los mismos fenómenos fuera de esta" (Berzelius, 1811: 257). De esta manera, él pretendía no sólo explicar el cambio químico sino el carácter electropositivo o negativo que tenían las sustancias en relación a otras con las cuales reaccionaba.

Berzelius, a partir de allí, atribuyó al oxígeno (que ya había sido descubierto por Lavoiser, quien hablaba de oxidación) la mayor electronegatividad y el carácter dual de otras sustancias como los no metales con respecto al oxígeno (electropositivo) o con respecto a metales (electronegativo). Sin embargo, es importante además considerar los trabajos de Liebig en relación a la deshidrogenación de los alcoholes en aldehídos, que definían la oxidación y reducción en función del hidrógeno. También los aportes de Lewis, ya que en el siglo XX, según la teoría electrónica de valencia, describe la reacción redox relacionada como una combinación de dos medias reacciones, incluida la transferencia de electrones, a la que posteriormente fue introducida el número de oxidación en relación a los cambios de oxidación. Finalmente, otro aspecto importante para la fundamentación y propuesta de la teoría electroquímica lo constituyen la relación proporcional entre el cambio químico, producido por la cantidad de electricidad, tal y como lo señaló Michael Faraday.

A través del libro Conversation on Chemistry de Jane Marcet (1806 - 1853) se muestra una visión historiográfica y didáctica, acerca de cómo evolucionó la teoría electroquímica en la primera mitad del S. XIX (Tabla 1), período fructífero para la comprensión del cambio químico y la propuesta de diferentes técnicas e instrumentos para su estudio (Marcet, 1917, 1932 y 1953). 


\begin{tabular}{|c|c|c|c|}
\hline Período & Evento Importante & $\begin{array}{l}\text { Conceptos relevantes sobre } \\
\text { electroquímica }\end{array}$ & $\begin{array}{l}\text { Conceptos relevantes sobre } \\
\text { la actividad química }\end{array}$ \\
\hline 1791 & Galvanismo & $\begin{array}{l}\text { Producción de la electricidad a } \\
\text { través de procesos naturales }\end{array}$ & $\begin{array}{l}\text { Relación de la química con } \\
\text { otras áreas del conocimiento } \\
\text { (Fisiología) }\end{array}$ \\
\hline 1800 & Construcción de la Pila de Volta & $\begin{array}{l}\text { Producción de la electricidad a } \\
\text { través de procesos artificiales }\end{array}$ & $\begin{array}{l}\text { Valor e importancia de los ins- } \\
\text { trumentos científicos }\end{array}$ \\
\hline 1806 & $\begin{array}{l}\text { Primera edición de Conversa- } \\
\text { tions on Chemistry }\end{array}$ & $\begin{array}{l}\text { Relación entre electricidad y } \\
\text { cambio químico }\end{array}$ & $\begin{array}{l}\text { Divulgación y enseñanza de la } \\
\text { química }\end{array}$ \\
\hline 1806 & $\begin{array}{l}\text { Conferencia de H. Davy Acerca } \\
\text { de algunos agentes químicos de } \\
\text { la electricidad }\end{array}$ & $\begin{array}{l}\text { Polarización de It sustancias. } \\
\text { Descomposición química a tra- } \\
\text { vés de la electricidad }\end{array}$ & $\begin{array}{l}\text { Evolución y desarrollo del con- } \\
\text { cepto de elemento química }\end{array}$ \\
\hline 1810 & $\begin{array}{l}\text { Lectura por Faraday de Conver- } \\
\text { sations on Chemistry }\end{array}$ & Estudio del cambio químico & $\begin{array}{l}\text { Contexto social y personal en la } \\
\text { construcción de conocimiento }\end{array}$ \\
\hline 1811 & Teoría Dualista de J.J. Berzelius & $\begin{array}{l}\text { Coexistencia de dos partes (po- } \\
\text { los) positiva y negativa en cual- } \\
\text { quier átomo. } \\
\text { Atracción y repulsión que ejer- } \\
\text { cen los polos sobre las sustan- } \\
\text { cias químicas (Afinidad quími- } \\
\text { ca) }\end{array}$ & $\begin{array}{l}\text { Distinción y debate sobre la } \\
\text { química orgánica e inorgánica }\end{array}$ \\
\hline 1836 & $\begin{array}{l}\text { Construcción de la Pila de Da- } \\
\text { niell }\end{array}$ & $\begin{array}{l}\text { Carácter oxidante y reductor de } \\
\text { las sustancias. } \\
\text { Reacciones redox }\end{array}$ & $\begin{array}{l}\text { Valor e importancia de los ins- } \\
\text { trumentos científicos }\end{array}$ \\
\hline 1853 & $\begin{array}{l}\text { Última versión de Conversations } \\
\text { on Chemistry }\end{array}$ & $\begin{array}{l}\text { Relación cuantitativa entre can- } \\
\text { tidad de sustancia y electrici- } \\
\text { dad. } \\
\text { Relación entre electricidad y } \\
\text { magnetismo }\end{array}$ & $\begin{array}{l}\text { Mujeres científicas en la cons- } \\
\text { trucción de conocimiento cien- } \\
\text { tífico }\end{array}$ \\
\hline 1817 & $\begin{array}{l}\text { Aparición de la Lectura sobre } \\
\text { los agentes químicos de la elec- } \\
\text { tricidad en Conversations on } \\
\text { Chemistry }\end{array}$ & $\begin{array}{l}\text { Relación entre galvanismo y } \\
\text { electrólisis a través de la Pila de } \\
\text { Volta }\end{array}$ & $\begin{array}{l}\text { Mujeres científicas en la cons- } \\
\text { trucción de conocimiento cien- } \\
\text { tífico }\end{array}$ \\
\hline 1832 & $\begin{array}{l}\text { Propuesta de las Leyes de Fara- } \\
\text { day }\end{array}$ & $\begin{array}{l}\text { Oxidación y reducción. } \\
\text { Conservación de cargas. } \\
\text { Cantidad de compuesto quí- } \\
\text { mico que se descompone es } \\
\text { proporcional a la cantidad de } \\
\text { electricidad utilizada }\end{array}$ & $\begin{array}{l}\text { Relación de la química con } \\
\text { otras áreas del conocimiento } \\
\text { (Matemática-Física) }\end{array}$ \\
\hline
\end{tabular}

Tabla 1. Evolución conceptual de la Teoría Electroquímica entre 1800 a 1853 
A partir de los antecedentes mencionados anteriormente, y con el propósito de promover la explicación científica a través de la historia de la ciencia, se diseñó una unidad didáctica para la enseñanza y aprendizaje de la teoría electroquímica (Camacho, Zanocco, Miranda, Astudillo \& Frías, 2010), la cual se describe en la tabla 2. En esta unidad didáctica se hizo explícito el interés por promover y desarrollar la explicación científica, así, cada una de las actividades propuestas, secuenciadas según el ciclo constructivista de aprendizaje (Sanmartí, 2000), estuvo orientada a construir explicaciones sobre la relación entre cambio químico-electricidad; igualmente, las estrategias desarrolladas con el estu- diantado consideró la lectura de fuentes primarias, la construcción de debates históricos, la elaboración de esquemas conceptuales enmarcadas en un contexto histórico y la réplica de experimentos. En el diseño de la unidad didáctica se hizo énfasis en 3 aspectos fundamentales: a) la construcción de conocimiento científico por parte de hombres y mujeres, enfatizando el aporte y trabajo de las mujeres en la actividad química; b) la construcción y diseño de instrumentos científicos y su valor en el desarrollo de la teoría electroquímica, particularmente acerca de la Pila de Volta y de Daniell y c) la divulgación de textos científicos, particularmente Conversations on Chemistry (Marcet, 1853).

\begin{tabular}{|c|c|c|c|}
\hline Fases & Actividad & Objetivo & Descripción \\
\hline $\begin{array}{l}\text { Exploración de ideas } \\
\text { previas }\end{array}$ & $\begin{array}{l}\text { Sobre la acción química } \\
\text { de la electricidad }\end{array}$ & $\begin{array}{l}\text { Identificar las ideas pre- } \\
\text { vias de los estudiantes so- } \\
\text { bre la relación entre elec- } \\
\text { tricidad y cambio químico. }\end{array}$ & $\begin{array}{l}\text { Lectura de la Conversa- } \\
\text { ción sobre los agentes } \\
\text { químicos de J. Marcet } \\
\text { (1853), para incorporar la } \\
\text { discusión sobre cómo se } \\
\text { produce la electricidad } \\
\text { y cuál es el rol de las mu- } \\
\text { jeres en el conocimiento } \\
\text { científico (Solsona, 2007). }\end{array}$ \\
\hline $\begin{array}{l}\text { Introducción de nuevos } \\
\text { conceptos }\end{array}$ & Debate Berzelius Vs Davy & $\begin{array}{l}\text { Explicar cómo se produ- } \\
\text { ce una reacción química } \\
\text { que origina electricidad } \\
\text { y cómo ocurre el proceso } \\
\text { de obtención de nuevas } \\
\text { sustancias, a partir de la } \\
\text { electricidad. }\end{array}$ & $\begin{array}{l}\text { Debate entre dos grupos, } \\
\text { quienes defendían una } \\
\text { posición y, posteriormen- } \\
\text { te, creaban consensos, a } \\
\text { fin de comprender cómo } \\
\text { se construye la ciencia } \\
\text { como actividad humana } \\
\text { de consensos y disputas. }\end{array}$ \\
\hline $\begin{array}{l}\text { Sistematización y Con- } \\
\text { clusiones }\end{array}$ & $\begin{array}{l}\text { ¿Qué competencias he- } \\
\text { mos desarrollado? }\end{array}$ & $\begin{array}{l}\text { Identificar y caracterizar } \\
\text { las competencias científi- } \\
\text { cas trabajadas y hacer una } \\
\text { revisión conceptual de los } \\
\text { principales aportes acerca } \\
\text { de la teoría electroquími- } \\
\mathrm{ca} \text {, }\end{array}$ & $\begin{array}{l}\text { Evaluación de las produc- } \\
\text { ciones de los estudiantes } \\
\text { a fin de autorregular sus } \\
\text { aprendizajes científicos. } \\
\text { Construcción de una línea } \\
\text { de tiempo con el fin de } \\
\text { evidenciar que el conoci- } \\
\text { miento científico evolucio- } \\
\text { na y es complejo, pues de- } \\
\text { pende de muchos factores } \\
\text { internos y externos. }\end{array}$ \\
\hline
\end{tabular}

Continúa 


\begin{tabular}{|c|c|c|c|}
\hline Fases & Actividad & Objetivo & Descripción \\
\hline Aplicación & $\begin{array}{l}\text { Construcción de la Pila de } \\
\text { Daniell }\end{array}$ & $\begin{array}{l}\text { Los estudiantes explica- } \\
\text { rán el funcionamiento de } \\
\text { la Pila de Daniel y el uso } \\
\text { de los diferentes tipos de } \\
\text { pilas }\end{array}$ & $\begin{array}{l}\text { Valor de la réplica de los } \\
\text { experimentos en la ense- } \\
\text { ñanza con el fin de com- } \\
\text { prender las reacciones } \\
\text { redox en las pilas y cono- } \\
\text { cer el sentido de la expe- } \\
\text { rimentación en la historia } \\
\text { de la electroquímica (Gra- } \\
\text { pí, 2006) }\end{array}$ \\
\hline
\end{tabular}

Tabla 2. Descripción de las actividades de la Unidad Didáctica de Teoría Electroquímica

\section{Resultados y consideraciones didácticas}

Los resultados que se presentan a continuación hacen parte de un análisis más profundo, a través de un estudio de casos colectivo (Camacho, 2010).

Para construir explicaciones científicas, el profesorado de química toma como punto de partida la definición de algunos conceptos preliminares, tales como cambio químico, electricidad, iones, cátodo, ánodo, sustancias electronegativas y electropositivas, polaridad, entre otros. Generalmente esto ocurre en el inicio de la secuencia didáctica, particularmente, en las actividades de exploración de ideas previas. Posteriormente, los docentes proponen establecer relaciones entre dichos conceptos, por ejemplo entre cargas eléctricas, iones, polarización en relación a las reacciones químicas, que permitían abordar la problemática plantada acerca de cómo se relaciona el cambio químico con la electricidad.

En algunos casos, las descripciones son trabajadas con mayor profundidad, en donde se hace énfasis en otorgar atributos o características a los conceptos nuevos que fueran coherentes con la teoría electroquímica y promover algunas relaciones entre los conceptos previos y los que se introducen, los cuales propone nuevamente definir o describir. De esta manera, la explicación científica consiste sólo en la descripción de conceptos, a partir de la teoría electroquímica y el establecimiento de algunas nuevas relaciones, las cuales incitan nuevamente a la descripción de los fenómenos relacionados con el cambio químico y la electricidad.

En suma, una secuencia inicial para construir la explicación científica tuvo como principal característica partir de una definición, seguido por descripciones entre los conceptos definidos y, luego, la elaboración de algunas conclusiones; en todas estas etapas la teoría electroquímica fue fundamental, así como la explicación de las ideas por parte de los estudiantes. La primera secuencia (Figura 2.) corresponde al trabajo inicial de la explicación científica, según señalan Sanmartí e Izquierdo (1998); Welsh (2002) y Jorba (2000).

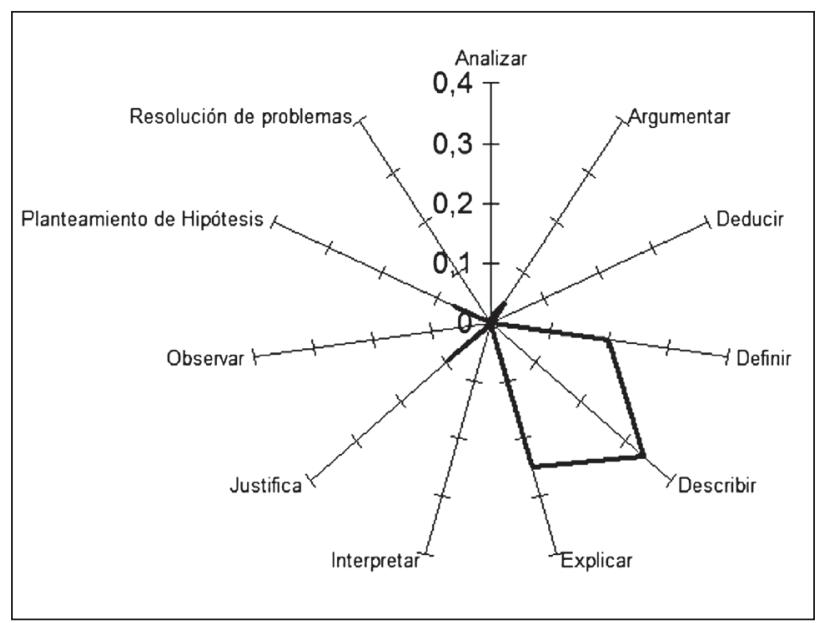

Figura 2. Primera secuencia para la construcción de la explicación científica

Fuente: Camacho, 2010: 361 
No obstante, en el proceso de construcción de explicaciones científicas también se percibió la descripción de fenómenos como la pila de Volta, pero, a diferencia de la secuencia anterior, se trató de orientar la relación entre los conceptos hacia la selección de información y la evaluación de la misma, de tal manera que se pudiera promover la elaboración de conclusiones, factibles de ser evaluadas. Bajo este panorama, el proceso para la construcción de explicaciones científicas escolares, corresponde a un proceso más complejo en donde -además de las descripciones- es necesaria la elaboración de razones o argumentos que podían ser enriquecidos por diversos aspectos teóricos o procedimentales de la teoría electroquímica. En esta segunda secuencia, las definiciones sólo se presentaron durante la actividad de exploración de ideas, posteriormente se propusieron conclusiones lo que llevó a que se generasen diferentes relaciones de causalidad entre los nuevos argumentos o razones, además de proponer espacios en el aula que permitían a sus estudiantes autorregularse y regular sus propias explicaciones.

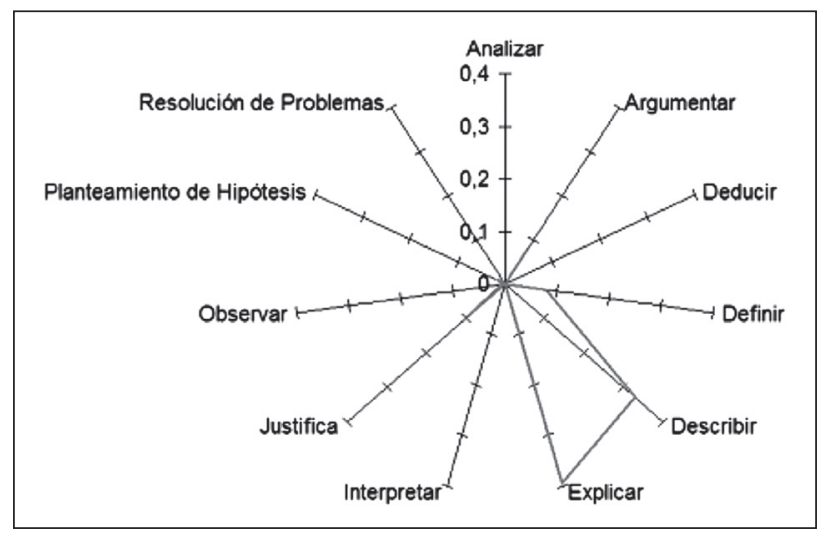

Figura 3. Segunda secuencia para la construcción de la explicación científica

Fuente: Camacho, 2010: 373

De esta manera, no sólo se abordó la parte conceptual de la competencia, sino que también se propusieron espacios metacognitivos para que el estudiantado reflexionara acerca de cómo eran sus propias explicaciones para mejorarlas. Estos nuevos aspectos, tal y como señalan Izquierdo y Chamizo (2005), permiten dar mayor consistencia a las conclusiones elaboradas. En virtud de lo anterior, esta segunda secuencia para construir la explicación científica se puede definir de la siguiente forma: definición, descripción, explicación, argumentación (Figura 3).

A través de esta experiencia es posible afirmar el aporte significativo de incorporar la historia de la ciencia para la construcción de explicaciones científicas. En este orden de ideas, se destaca que en este proceso es importante y necesario plantear "situaciones científicas escolares problematizadoras" (Camacho \& Quintanilla, 2008), en donde se intencionan diferentes aspectos relacionados con la competencia científica, entre ellos, los espacios de discusión teórica y reflexión metacognitiva, actividades de debate y prácticas experimentales que involucran el pensamiento, la experiencia y el lenguaje de las personas competentes. Actividades como la lectura del texto adaptado de Jane Marcet o el debate entre H. Davy y J.J. Berzelius permitieron un mayor protagonismo al estudiantado, promoviendo su participación como personas epistemológicamente activas y la construcción de la competencia desde sí mismos con regulación del profesorado, promoviendo más grados de libertad en sus intervenciones, hasta el momento final de la secuencia en donde son los mismos estudiantes quienes construyen su propia explicación científica. Este aspecto no sólo es muy importante para la construcción de conocimiento científico escolar, sino que además es significativo para el estudiante ya que le ayuda a construir su competencia científica.

[...] yo creo porque, por ejemplo, si quizás nos pasarán la materia de una forma más estricta, quizás no lo entenderíamos, pero haciendo actividades y cosas así que a nosotros nos llamen la atención es mucho más fácil de aprender [...] a mí me gusta más, yo creo que uno aprende mejor cuando tiene que exponer, por ejemplo cuando uno primero hace una redacción del trabajo que te dan y luego, exponer, porque así por una parte le queda porque se lo tiene que aprender para poder exponerlo, pero por otra parte lo que tus compañero te escuchan también te queda [...] cuando tuvimos que hacer un escrito porque ahí fue cuando trabajamos más, la argumentación porque es mucho más fácil cuando te dan un texto, y ahí uno puede explicar, porque si uno quiere explicar algo lo más fácil es dar argumentos, argumentos, hasta podemos hacer un debate con los que 
piensan una cosas y los que piensan otra cosa. (Entrevista focalizada, estudiante 1).

Otras instancias importantes en la construcción de la explicación científica, la constituye la promoción de actividades orientadas a la metacognición y autorregulación, en donde se promuevan actividades que permitan al estudiantado construir conclusiones y evaluarlas en el marco de la teoría científica. Dicho aspecto permitió la autorregulación del estudiantado y la orientación hacia la construcción de la explicación, así como conocer explícitamente y evaluar qué es la explicación como competencia científica.

La actividad de aplicación, que en este caso consistió en la construcción de la Pila de Daniell, demostró una gran oportunidad de poner en juego el tipo de conclusiones construidas y así dar mayor robustez a la explicación científica del fenómeno de la acción química de la electricidad. Esta oportunidad, además, sugirió la construcción de nuevas conclusiones que permitieron proponer argumentos nuevos sobre lo que podría ocurrir o no. Así, se señala que las actividades experimentales y de aplicación son muy relevantes en la construcción de la explicación científica y que también favorecen la relación entre los contenidos conceptuales como reacciones de óxido reducción, transferencia electrónica, carga en el ánodo y el cátodo, como los aspectos procedimentales de la teoría electroquímica, predecir productos y magnitudes en una reacción de óxido reducción.

La promoción de la explicación como competencia científica supone repensar los propósitos de la enseñanza-aprendizaje de la química escolar, a fin de transformar la práctica docente. En virtud de lo cual se hace necesario establecer actividades secuenciadas, en relación a dichos propósitos e instalar dispositivos de evaluación. Los resultados en la manera en que se promovió y desarrolló la explicación científica permiten evidenciar que esta nueva fundamentación teórica supone elementos que enriquecen la competencia y que orientan hacia una nueva manera de mirar la actividad química escolar.
Por último se señala que, además de promover la explicación científica con respecto a la teoría electroquímica, esta competencia abordó otros aspectos en relación a la historia y naturaleza de la ciencia que contribuyeron positivamente a la propia construcción de la competencia por parte del estudiantado.

“[...] viendo como el desarrollo y el aporte de cada uno de ellos a la electroquímica en este caso que era lo que estábamos viendo, viendo cada una de las cosas, como los métodos que usaban ellos o los distintos tipos de experimentos que usaban, entonces era un complemento de lo que era la historia y la electroquímica. Eran las cosas más entretenidas y no sólo fórmulas, fórmulas y fórmulas [...] sí, aprender lo que son expectativas de vida, porque había mucho científico que a lo mejor en su momento lo juzgaban, entonces lo que era la Historia de la Ciencia no sólo servía para lo que era electroquímica, sino también podía llegar a servir, depende de la historia de cada científico, para el diario vivir, así como plantearse metas y poder cumplirlas. Entonces, esas cosas nos enseñaban, a parte de lo que era electroquímica, un montón de cosas más, como argumentar. Ellos, a lo mejor, tuvieron que luchar contra distintos postulados, de repente hasta entre ellos mismos por cosas que no compartían, entonces la argumentación, los puntos de vista, el no dejarse llevar de repente, cosas así. (Entrevista focalizada. Estudiante 2)

\section{Bibliografía}

Berzelius, J. J. (1811). “Suite des expériences sur les proportions déterminées, d’après lesquelles les éléments de la nature inorganique s'unissent". En Annales de Chimie, núm. 79, p. 233-264. Bliss, Monk y Ogborn, 1983.

Camacho, J. (2005). "Ley Periódica. Una reflexión didáctica desde la historia de las Ciencias", [tesis de Magíster en Docencia de la Química]. Facultad de Ciencia y Tecnología, Departamento de Química. Universidad Pedagógica Nacional. Bogotá D.C., Colombia.

Camacho, J. \& Martínez, N. (2003). "Calor y temperatura: Una precisión histórico epistemológica para el estudio de la transposición didáctica", [tesis de Licenciatura en Docencia de la Química]. Facultad de Ciencia y Tecnología, Departamento de Química. Universidad Pedagógica Nacional. Bogotá D.C., Colombia. 
Camacho, J., Gallego, R. \& Pérez, R. (2007). “Ley Periódica. Una reflexión didáctica desde la historia de las ciencias". En Revista Educación en Química, vol. XVIII, núm. 24, pp. 278-288.

Camacho, J. \& Quintanilla, M. (2008). "Resolución de problemas científicos desde la Historia de la Ciencia. Retos y desafíos para promover competencias cognitivo lingüísticas en la química escolar". En Ciência \& Educação, vol. 14, núm. 2, pp. 197-212.

Camacho, J., Zanocco, G., Miranda, O., Astudillo, C. \& Frías, A. (2010). “Teoría electroquímica en la educación media. Una propuesta didáctica fundamentada en la Historia de la Ciencia. En Quintanilla, M., Daza, S. \& Merino, C. (Comp.), Su contribución a las competencias de pensamiento científico, vol. III. Santiago de Chile, Chile.

Davies, A. J. (1991). "A model approach to teaching redox". En Education in Chemistry, vol. 28, pp. 135-137.

De Jong, O. \& Treagust, D. (2002). “The teaching and learnig of electrochemical". En Gilbert, et al. (eds.), Chemical Education. Towards Research based practice. pp. 317-337. Netherlands: Kluwer Academia Publishers.

De Jong, O., Acampo, J. \& Verdonk, A. (1995). "Problems in teaching the Tepic of redox reactions: actions and conceptions of chemistry teachers". En Journal of Research in Science Teaching, 32, pp. 1097-1110.

Giere, R. (1992). La explicación de la ciencia. Un acercamiento cognitivo. México: Consejo Nacional de Ciencia y Tecnología.

Gooday, G., et al., (2008). “Does science education need the history of science?” En Isis, vol. 99, pp. 322-330.

Grapí, P. (2006). "L'electrificació de la química al Segle XVIII. Una xarxa de guspires. Actes d'història de la ciència i de la tècnica". En Nova Ėpoca, vol. 1, núm. 1, pp. 65-74.
Grapí, P. i Vilumara, P. (2008). 'L' electrificació del camvi químic. Els inicis al primer quart del segle XIX”. En Educació Químic, vol. 1, pp. 51-57.

Griffths, A. K. (1994). "A critical análisis and synthesis of research on students ' chemistry misconceptions". En Schimidt (ed). Problem solving and misconceptions in chemistry and physics, pp. 70-99. Hong Kong: ICASE.

Gubler, J. \& Williamson, A. (2009). "Resultados de los estudiantes chilenos en la prueba PISA Ciencias 2006: una mirada a sus competencias". En Cariola, et al., (Coords). ¿Qué nos dice PISA sobre la educación de los jóvenes en Chile? Nuevos análisis y perspectivas sobre los resultados en PISA 2006. pp. 197-237. MINEDUC: Unidad de Curriculun y Evaluación.

Gutiérrez, A. (2008). "La evaluación de las competencias científicas en PISA: perfiles en los estudiantes iberoamericanos". En Alambique Didáctica de las Ciencias Experimentales, núm. 57, pp. 23-31.

Hempel, C. G. (1965). Aspects of scientific explanation and other essays in the philosophy of scien$c e$. New York: Free Press.

Izquierdo, M. \& Adúriz-Bravo, A. (2009). "A Research-informed instructional unit to teach the nature of science to pre-service science teachers". En Science \& Education vol.18, núm. 2, pp. 1177- 1192.

Izquierdo, M. (2004). "Un nuevo enfoque de la enseñanza de la química: contextualizar y modelizar". En The Journal of the Argentin Chemical Society, vol. 92, núm. 4/6, pp.115-136.

Jorba, J. (1998). "La comunicació i les habilitats cognitivolingüístiques". En Jorba, J., et al. (eds.). Parlar i escriure per aprendre. Barcelona: ICE-UAB.

Marcet, J. (1853). "Conversations on chemistry in which the elements of that science are familiarly explained and illustrated by expe- 
riments", vol. 1 On simple bodies. London: Longman, Brown, Green \& Longmans.

Mineduc, (2009). Ajuste curricular en el sector de Ciencias Naturales. Santiago: Unidad de Currículo y Evaluación.

Níaz, M. \& Chancón, E. (2003). “A conceptual change teaching strategy to facilitate high school students' understanding of electrochemistry". En Journal of Science Education and Techonology, vol. 12, núm. 2, pp. 129-134.

OCDE (2006). PISA 2006. Marco de la evaluación. Conocimientos y habilidades en Ciencias, Matemática y Lectura. París, Francia: OCDE.

OCDE (2010). PISA 2009 Results: What Students Know and Can do. Student performance in reading, mathematics and Science. vol. 1, [en línea]. Disponible en: http://dx.doi. org/10.1787/9789264091450-en [recuperado el 1 de Abril de 2011].

Özkaya, A. (2002). "Conceptual difficulties experienced by prospective teachers in electrochemistry: half-cell potential, cell potential, and chemical and electrochemical equilibrium in galvanic cells". En Journal of Chemical Education, vol. 79, núm. 6, pp. 735-738.

Quintanilla, M., et al., (2009). Desarrollo, caracterización y validación de un modelo de evaluación de competencias de pensamiento científico en estudiando de enseñanza media basado en el enfrentamiento a la resolución de problemas para promover aprendizajes de calidad. Proyecto FONDECYT 1095149.

Quintanilla, M., et al., (2010). "Resolución de problemas científicos escolares y promoción de competencias de pensamiento científico. ¿Qué piensan los docentes de química en ejercicio?". En Enseñanza de las ciencias, vol. 28, núm. 9, pp. 185-198.

Rosenberg, A. (2000). Philosophy of science: a contemporary introduction. Routledge, London.

Sanmartí, N. (2000), "El diseño de unidades didácticas". En Canal, P., Perales, J. (edits.). Didáctica de las Ciencias Experimentales. Alcoy: Marfil. pp. 239- 266.

Sanmartí, N. e Izquierdo, M. (1998). "Enseñar a leer y escribir textos en ciencias". En Jorba, J., Gómez, L. \& Prat, A. (edits.). Hablar y escribir para aprender. pp. 181-199. España, Bellaterra: ICE de la UAB.

Solsona, N. (2007). "Las Mujeres en la Historia de la Ciencia”. En Quintanilla \& Adúriz-Bravo, (Ed), Enseñar ciencias en el nuevo milenio. Retos $y$ desafíos. pp. 37-63. Santiago, Chile: Pontificia Universidad Católica de Chile.

Treviño, E., Donoso, F. \& Bonhomme, M. (2009). “Cómo las escuelas chilenas pueden mejorar el aprendizaje en Ciencias?”. En Cariola, et al., (coords). ¿Qué nos dice PISA sobre la educación de los jóvenes en Chile? Nuevos análisis y perspectivas sobre los resultados en PISA 2006 (pp.71-104) MINEDUC: Unidad de Curriculum y Evaluación.

Welsh, S. (2002). "Advice to a new science teacher: the importance of establishing a theme in teaching scientific explanations". En Journal of Science Education and Technology, vol. 11, núm. 1, pp. 93-96. 